ARTICLE

Received 31 Aug 2016 | Accepted 5 Jan 2017 | Published 22 Feb $2017 \quad$ DOl: 10.1038/ncomms14509 OPEN

\title{
Allograft inflammatory factor 1 is a regulator of transcytosis in $M$ cells
}

\author{
Sari Kishikawa ${ }^{1,2, \star}$, Shintaro Sato ${ }^{1,3,4,5,6, \star}$, Satoshi Kaneto ${ }^{1}$, Shigeo Uchino ${ }^{7} \dagger$, Shinichi Kohsaka ${ }^{7}$, Seiji Nakamura ${ }^{2}$ \\ \& Hiroshi Kiyono $1,3,4,8$
}

$M$ cells in follicle-associated epithelium (FAE) are specialized antigen-sampling cells that take up intestinal luminal antigens. Transcription factor Spi-B regulates M-cell maturation, but the molecules that promote transcytosis within $M$ cells are not fully identified. Here we show that mouse allograft inflammatory factor 1 (Aif1) is expressed by $\mathrm{M}$ cells and contributes to $\mathrm{M}$-cell transcytosis. FAE in Aif1 ${ }^{-/-}$mice has suppressed uptake of particles and commensal bacteria, compared with wild-type mice. Translocation of Yersinia enterocolitica, but not of Salmonella enterica serovar Typhimurium, leading to the generation of antigen-specific IgA antibodies, is also diminished in Aif1-deficient mice. Although $\beta 1$ integrin, which acts as a receptor for $Y$. enterocolitica via invasin protein, is expressed on the apical surface membranes of $M$ cells, its active form is rarely found in Aif $1^{-/-}$mice. These findings show that Aif1 is important for bacterial and particle transcytosis in $M$ cells.

\footnotetext{
${ }^{1}$ Division of Mucosal Immunology, The Institute of Medical Science, The University of Tokyo, Tokyo 108-8639, Japan. ${ }^{2}$ Section of Oral and Maxillofacial Oncology, Division of Maxillofacial Diagnostic and Surgical Sciences, Faculty of Dental Science, Kyushu University, Fukuoka 812-8582, Japan. ${ }^{3}$ International Research and Development Center for Mucosal Vaccines, The Institute of Medical Science, The University of Tokyo, Tokyo 108-8639, Japan. ${ }^{4}$ Core Research for Evolutional Science and Technology, Japan Science and Technology Agency, Tokyo 102-0076, Japan. ${ }^{5}$ Mucosal Vaccine Project, BIKEN Innovative Vaccine Research Alliance Laboratories, Research Institute for Microbial Diseases, Osaka University, Osaka 565-0871, Japan. ${ }^{6}$ Mucosal Vaccine Project, BIKEN Center for Innovative Vaccine Research and Development, The Research Foundation for Microbial Diseases of Osaka University, Osaka 565-0871, Japan. ${ }^{7}$ Department of Neurochemistry, National Institute of Neuroscience, Tokyo 187-8502, Japan. ${ }^{8}$ Department of Immunology, Graduate School of Medicine, Chiba University, Chiba 260-8670, Japan. * These authors contributed equally to this work. † Present address: Department of Biosciences, School of Science and Engineering, Teikyo University, Tochigi 320-8551, Japan. Correspondence and requests for materials should be addressed to S.S. (email:

shinta@biken.osaka-u.ac.jp) or to H.K. (email: kiyono@ims.u-tokyo.ac.jp).
} 
ntestinal tissues are continuously exposed to the outside environment. The epithelium covering the digestive tract is the barrier to invasion by gut pathogenic bacteria and interface to mutual interaction with commensal microbiota. Therefore, intestinal epithelial cells (IECs) are equipped with a variety of immunological, physiological and chemical barrier features to maintain the balance between surveillance or elimination and symbiosis, and thus create intestinal homeostasis ${ }^{1-4}$. These features include innate antigen-recognition receptors such as Toll-like receptors, along with acquired immunity (for example, in the form of secretory $\operatorname{IgA}$ ), tight junction molecules (for example, occludin), and production of antimicrobial peptides (for example, defensin), cytokines, chemokines and mucins ${ }^{4}$. Offensive and defensive interactions between host and bacteria influence the induction and regulation of the antigen-specific mucosal immune responses. To induce antigenspecific immune responses against orally encountered antigens, the mucosal immune system is functionally organized into inductive tissues such as Peyer's patches (PPs) and effector tissues such as the lamina propria ${ }^{5,6}$. PPs are well-characterized inductive tissue in the small intestine and are covered by follicle-associated epithelium (FAE) ${ }^{6}$. FAE contains microfold (M) cells, which are specialized antigen-sampling cells that actively take up foreign antigens from the intestinal luminal side into PPs for the initiation of antigen-specific humoral and cellular immune responses ${ }^{7}$. M cells have two unique structural characteristics; they have irregular, short microvilli on their apical side that distinguish them from neighbouring columnar epithelial cells with tall and dense microvilli, and they have a pocket structure holding antigen-presenting cells such as macrophages, $\mathrm{B}$ cells, and dendritic cells on their basolateral side ${ }^{8-11}$. This unique morphology is considered to contribute to their active antigen uptake and the subsequent transcytosis of antigens from the intestinal lumen to antigen-presenting cells in PPs, resulting in the initiation of antigen-specific mucosal immune responses ${ }^{7,12}$.

Glycoprotein 2 (GP2) has been identified as a specific marker of mature M cells; it contributes to the uptake of Salmonella enterica serovar Typhimurium by recognising the bacterial flagellar protein $\mathrm{FimH}^{13,14}$. In addition, cellular prion protein on the M-cell surface has been reported to be an invasive receptor for Brucella abortus ${ }^{15}$. Furthermore, Spi-B is a pivotal transcription factor for the development and function of $M$ cells, including the induction of GP2 (refs 16-18). Despite these advances, we still have a limited understanding of the molecular basis of M-cell development, maturation, and function (for example, transcytosis).

To further understand the immunobiological uniqueness of $\mathrm{M}$ cells, we previously performed a DNA microarray analysis to search for new molecules (our unpublished data) and identified several unique candidate genes specifically associated with $\mathrm{M}$ cells. From this assay, we proposed allograft inflammatory factor 1 (Aif1) as a candidate protein that is encoded by one of these genes and is preferentially produced by $\mathrm{M}$ cells. Aif1, which is also known as ionized calcium-binding adapter molecule 1 (Iba1), is a cytoplasmic protein with EF-hand calcium-binding domains and was first observed in the macrophages of rat heart allografts under chronic rejection ${ }^{19}$. Aifl is highly expressed in the monocytic lineage, including macrophages and microglia, and is involved in phagocytosis and the formation of membrane ruffling through Racl, which belongs to the Rho-family of GTPases ${ }^{20,21}$. In addition, when EF-hand-domain-truncated or point-mutated Aif1 is overexpressed in the microglial cell line MG5, the cells cannot form membrane ruffling through actin remodelling to catch foreign antigens such as zymosan during phagocytosis ${ }^{21,22}$. However, it has been suggested that, in vascular smooth muscle cells, Aif1-Racl signalling is involved in cell migration and actin polymerisation rather than membrane ruffling ${ }^{23}$. The precise function of Aif1 in $\mathrm{M}$ cells is unknown. Here we generate Aif1-deficient mice to investigate in vivo role of Aif1 in M cells. Aif1 deficiency does not affect the development and fundamental ultrastructure of $\mathrm{M}$ cells. However, uptake of particles, commensal and pathogenic bacteria by $M$ cells is severely impaired in Aif1-deficient mice. Our findings suggest that M-cell-intrinsic Aif1 plays an important role in antigen uptake and transcytosis function of $\mathrm{M}$ cells.

\section{Results}

Specific expression of Aif1 by $\mathbf{M}$ cells. To shed further light on M-cell-specific molecules, we performed a DNA microarray analysis by using RNA prepared from the FAE of $S p i b^{+/+}$and $S p i b^{-/}$mice, because previous studies by ourselves and others had shown that Spi-B deficiency resulted in a substantial reduction in M-cell development ${ }^{16-18}$. We therefore used FAE from the Spib ${ }^{+1+}$ mice as normal FAE and that from the Spib ${ }^{-1-}$ mice as M-cell-deficient FAE. From this analysis we identified several candidate genes, the expression of which was identified as M-cell specific and Spi-B dependent (unpublished data). Here we focused on Aif1, because Aif1 is involved in phagocytosis in macrophages through modification of actin bundling ${ }^{20,21}$ and in migration of vascular smooth muscle cells through the induction of actin polymerization ${ }^{23}$.

First, we examined M-cell-specific expression of Aif1 by quantitative PCR analysis of various IECs, including FAE, which were isolated from Spi-B-deficient mice and littermate controls. In control mice, Aif1 mRNA was highly expressed in haematopoietic cell lineages prepared from PPs, as reported previously (Fig. 1a) ${ }^{21}$. In fact, CD11c-positive cells in PPs and the lamina propria also expressed Aif1 (Supplementary Fig. 1). Aif1 was also highly expressed in FAE, but not in other small or large intestinal epithelial cells (Fig. 1a), though its level was lower than other known M-cell markers such as Gp2, Spib and Ccl9 (Supplementary Fig. 2). Expression of Aif1 mRNA in FAE was severely defective in Spi-B-deficient mice. These results suggested that, among the various types of IECs, Aif1 expression might be specific for $M$ cells. Expression of Aif1 in haematopoietic cells prepared from PPs was intact in Spi-B-deficient mice, further supporting the specificity of Aif1 expression by $\mathrm{M}$ cells and its dependence on Spi-B (Fig. 1a).

We next used whole-mount staining of PPs to investigate the expression of Aif1 protein in M cells. PPs prepared from wildtype mice were stained with anti-Aif1 and anti-GP2 antibodies, because GP2 positivity in FAE is definitive of mature $\mathrm{M}$ cells ${ }^{13,14}$. In wild-type mice, although it seemed that there were some GP2- or Aif1-single positive cells because the fluorescein signals of both GP2 and Aif1 are different in each cell, most of the Aif1-positive cells located in the FAE of PPs were co-stained with anti-GP2 antibody (Fig. 1b). $Z$-stack images also showed that the expression of Aif1 was restricted in GP2-positive cells (Supplementary Fig. 3). However, Aif1 expression, like GP2 expression, was absent in the FAE of PPs prepared from $S_{p i b}{ }^{-/}-$mice (Fig. 1c). On the other hand, consistent with the mRNA expression (Fig. 1a), production of Aif1 was detected in the sub-epithelial dome and lamina propria regions, even in Spi-B-deficient PPs (Supplementary Fig. 4). These data suggest that Aif1 is a newly identified M-cell-specific molecule, the expression of which depends on the presence of Spi-B.

Generation and characterisation of Aif1-deficient mice. To investigate the functional role of Aif1 in $\mathrm{M}$ cells in vivo, we generated Aif1-deficient $\left(A i f 1^{-/-}\right)$mice (Supplementary 
a

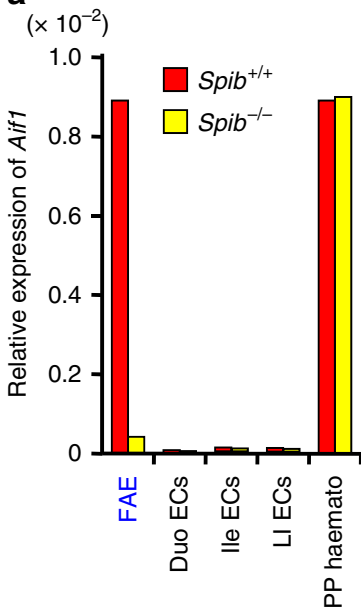

b
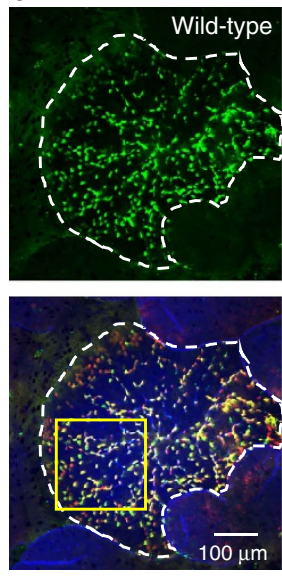
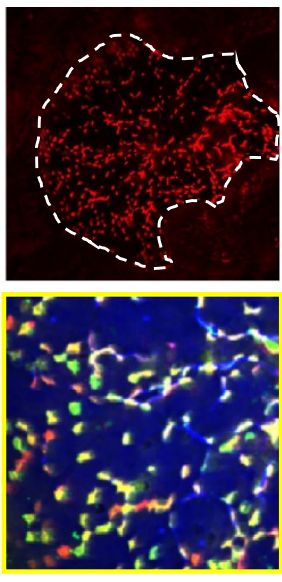

C

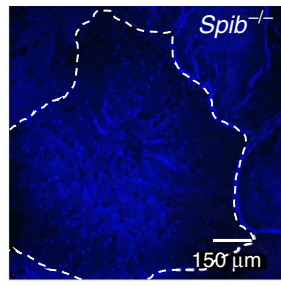

GP2

Aif1

F-actin

Figure 1 | Specific expression of Aif1 in GP2-positive $\mathbf{M}$ cells among IECs. (a) Relative Aif1 expression in indicated cell populations from Spib $+/+$ and Spib ${ }^{-/-}$mice. Each result was normalized against the expression of glyceraldehyde 3-phosphate dehydrogenase (Gapdh) and is representative of three independent experiments. (b,c) Whole-mount staining of GP2-positive M cells and Aif1. Peyer's patches (PPs) prepared from wild-type C57BL/6J (b) and Spi-B-deficient mice (c) were stained with anti-GP2 antibody (green), anti-Aif1 antibody (red) and phalloidin (blue). The yellow box region in (b, bottom left) was magnified to produce the image in (b, bottom right). Dotted lines indicate the periphery of the FAE region. Data are representative of three independent experiments. Duo, duodenal; ECs, epithelial cells; FAE, follicle-associated epithelium, haemato, haematopoetic cells; lle, ileum; LI, large intestine.
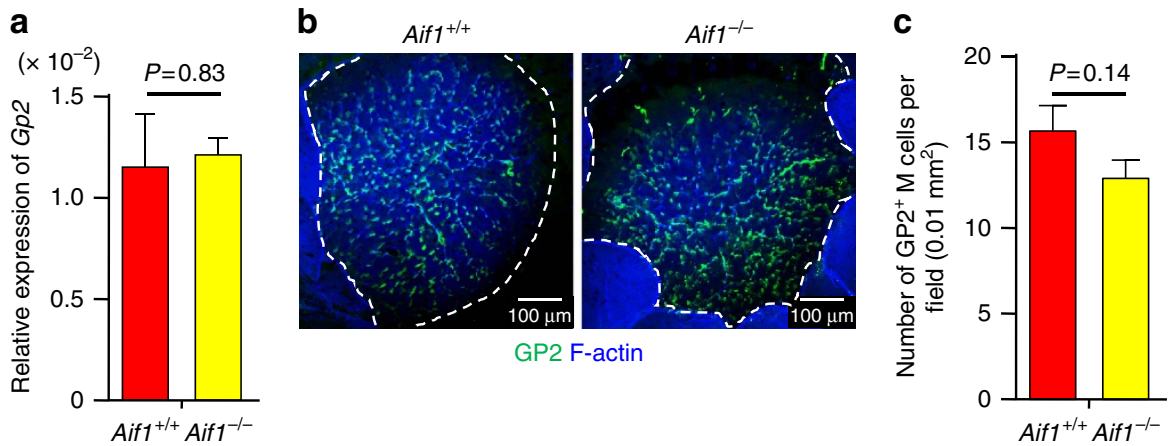

Figure 2 | Normal M-cell numbers in Aif1-deficient mice. (a) Relative expression of Gp2 in FAE from Aif $1^{+/+}$and Aif1 ${ }^{-/-}$mice. Each result was normalized against the expression of Gapdh. Data are shown as means \pm s.e.m. ( $n=2$ per genotype) from one experiment representative of two independent experiments. $P$ value was determined with Student's $t$-test. (b) Whole-mount staining of GP2-positive M cells in the FAE of Aif1 $+/+$ and Aif $1^{-/-}$mice. The number of GP2-positive cells found in each $0.01-\mathrm{mm}^{2}$ field was counted to provide the data in $\mathbf{c}$. Dotted lines indicate the periphery of the FAE region. Data are representative of three independent experiments. (c) M cells were counted as GP2-positive cells, and the average number of $M$ cells found in each $0.01 \mathrm{~mm}^{2}$ field is shown as the mean \pm s.e.m. $(n=7$ per genotype). $P$ value was determined with Student's $t$-test.

Fig. 5a). Aif $1^{-/-}$mice were born in the expected Mendelian ratio and showed no developmental abnormalities. Immunoblot analysis confirmed that Aif1 expression was abolished in Aif1 $1^{-/}$bone-marrow (BM) cells and splenocytes (Supplementary Fig. 5b). Furthermore, Aif1 expression in FAE of PPs was not detected by whole-mount staining (Supplementary Fig. 5c).

Next, we checked whether there were any differences in FAE Gp2 expression between wild-type and Aif1-deficient mice. Quantitative PCR analysis revealed that expression levels of Gp2 mRNA in FAE were comparable in control and Aif1 -/mice (Fig. 2a). In addition, almost the same number of GP2-positive cells (that is, mature M cells) per field of FAE was observed in both types of mice (Fig. 2b,c). These data suggest that Aif1 deficiency did not affect M-cell maturation.

M-cell morphology is unaffected by Aif1-deficiency. As indicated above, Aifl deficiency did not influence the production of GP2 by M cells. In our next experiment, we examined whether
Aif1 was involved in M-cell morphology. Scanning electron microscopic analysis revealed that the apical surface morphology (short, irregular microvilli formation) of $\mathrm{M}$ cells in Aif1 ${ }^{-7}-$ mice was similar to that in control mice (Fig. 3a). Consistent with the findings on GP2 positivity, the number of indented cells (which are considered to be $\mathrm{M}$ cells ${ }^{16,24}$ ) per field of FAE in Aif1 ${ }^{-/-}$ mice did not differ significantly from that in control mice (Fig. 3b). Furthermore, the presence of microvilli and a pocket structure enfolding lymphocytes was observed in the FAE region of Aif1-deficient mice, in the same way as in wild-type mice (Fig. 3c). In addition, the endocytic compartments found at the apical side of $\mathrm{M}$ cells were observed in Aif $1^{-/-}$mice, although the size and number of endosomes were slightly smaller than those found in control mice (Fig. 3d). These data suggest that Aif1 is not associated with the morphology of $\mathrm{M}$ cells.

Aif1 is important for antigen-uptake function of $M$ cells. Although Spi-B has been shown to be a key transcription factor 
a

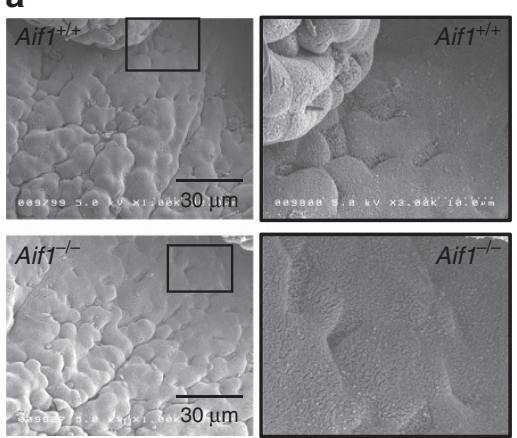

C

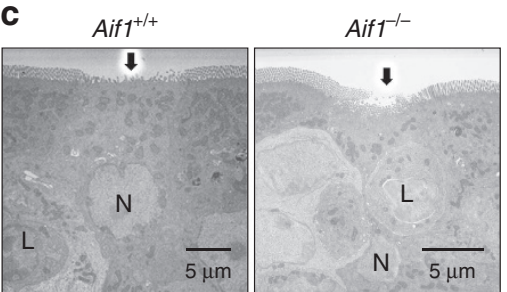

b

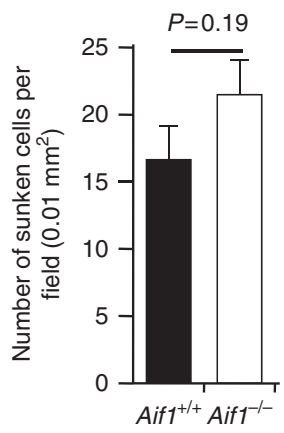

d

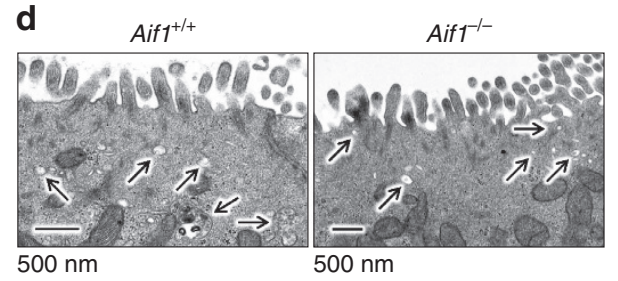

Figure 3 | Ultrastructure of $\mathbf{M}$ cells in Aif1-deficient mice. (a) Scanning electron microscopic images of FAE from control and Aif1 ${ }^{-/-}$mice. Right panels are magnified images of the boxed regions in the left panels. (b) $M$ cells were counted as sunken cells, and the average number of $M$ cells found in each $0.01 \mathrm{~mm}^{2}$ field is shown as the mean \pm s.e.m. of one experiment representative of two independent experiments $\left(\right.$ Aif $1^{+/+}, n=9 ;$ Aif $\left.1^{-/-}, n=10\right)$. $P$ value was determined with Student's $t$-test. (c) Transmission electron microscopic images of FAE from control and Aif $1^{-/}{ }^{-}$mice. Arrows indicate $M$ cells. Data are representative of three independent experiments. N, nucleus; L, lymphocyte. (d) Magnified transmission electron microscopic images of the apical side of $\mathrm{M}$ cells. Arrows show endosomes. Data are representative of three independent experiments.

associated with $\mathrm{M}$-cell development ${ }^{16-18}$, very little is known about the molecular mechanisms of the M-cell's antigensampling system. We therefore next examined whether Aif1 contributed to the antigen-uptake function of $M$ cells. First, we compared the uptake of fluorescent particles $(200 \mathrm{~nm}$ in diameter) between control and Aif1 $1^{-/-}$mice. Two hours after oral administration of these particles, PPs from the duodenum were removed and the number of particles in each PP was counted under a fluorescence microscope. The PPs of Aif $1^{-/-}$mice took up dramatically and significantly fewer particles than did the PPs of Aif1 ${ }^{+}+$mice (Fig. 4a). We then performed the same assay by using fluorescencelabelled Lactobacillus reuteri as larger particulate antigens $(0.7$ to $1.0 \times 2.0$ to $3.0 \mu \mathrm{m}$ in size, ref. 25$)$; this bacterium is one of the major commensals found in the mouse small intestine ${ }^{26}$. The numbers of bacteria found in FAE and inside the PPs were significantly smaller in $A i f 1^{-/-}$mice than in control Aif $1^{+1+}$ mice (Fig. 4b).

Next, we used Yersinia enterocolitica and S. Typhimurium to examine the influence of Aif1 deficiency on M-cell-targeted invasion of PPs by intestinal pathogenic bacteria. Wild-type control and Aif $1^{-1-}$ mice were orally given $1 \times 10^{9}$ colonyforming units (CFUs) of Y. enterocolitica or $1 \times 10^{8}$ CFUs of $S$. Typhimurium. Twenty-four hours after oral administration of the pathogens, PPs were isolated from the ileum, weighed, homogenized, and diluted in PBS. Each diluted sample was plated on $Y$. enterocolitica-selective agar, or on LB agar plates containing $25 \mu \mathrm{g} \mathrm{ml}^{-1}$ nalidixic acid to select $S$. Typhimurium, before overnight incubation at the appropriate temperatures (see Methods section). We then counted the numbers of colonies. Surprisingly, in a result similar to that for the particle uptake, the number of CFUs of $Y$. enterocolitica found in the PPs was significantly lower in Aif1 $1^{-/-}$mice than in wild-type mice (Fig. 5a). In contrast, the number of $S$. Typhimurium found in the PPs did not differ significantly between control and Aif $1^{-/-}$mice (Fig. 5b).
As shown above (Fig. 2b), the expression of GP2 was not impaired in Aif1-deficient mice. Given that GP2 recognizes the outer membrane protein FimH on $S$. Typhimurium and contributes to efficient transcytosis of such bacteria by $\mathrm{M}$ cells ${ }^{14}$, the presence of GP2 at the apical surfaces of M cells upon uptake of $S$. Typhimurium might be the reason why Aif1-deficient mice showed no decline in the number of $S$. Typhimurium in the PPs. To investigate this hypothesis, we next prepared and used a FimH-deletion mutant of $S$. Typhimurium $(\Delta$ Fim $H)$. In wild-type mice, the number of $\triangle$ FimH S. Typhimurium entering the PPs was smaller than the number of parental FimH-positive bacteria entering (Fig. 5c,b). Translocation of $\Delta$ FimH S. Typhimurium compared with parental FimH-positive bacteria was also diminished in Aif $1^{-/}$mice, but contrary to our speculation, this effect was no more than in wild-type mice (Fig. 5c). Salmonella spp. invasion induces membrane ruffling by their type-three secretion system (TTSS) ${ }^{27}$. In the case of Yersinia spp. invasion, membrane ruffling is not needed and does not occur ${ }^{2 f, 28}$. Therefore, we next examined the uptake of the $\triangle i n v A$ mutant of $S$. Typhimurium, which lacks TTSS and cannot induce membrane ruffling ${ }^{27}$. Because of this lack of TTSS, the uptake number of $\triangle i n v A$ mutant organisms was severely reduced, even in wild-type control mice, but it was more severely reduced in Aif $1^{-/-}$mice (Fig. 5d; $P=0.016$ versus wild-type mice). Taken together, these results suggest that Aif1 is associated with actin remodelling occurring on $Y$. enterocolitica invasion, but not with membrane ruffling, which is induced by, and needed for, invasion of $S$. Typhimurium.

M-cell-intrinsic Aif1 is important for mucosal immunity. Aif1-deficient mice showed decreased M-cell-mediated uptake of particles and translocation of Y. enterocolitica. Aif1 expression has been reported so far in microglia and macrophages ${ }^{21,29}$. Therefore, it was also important to investigate whether Aif1, which is produced in macrophages, dendritic cells, and 

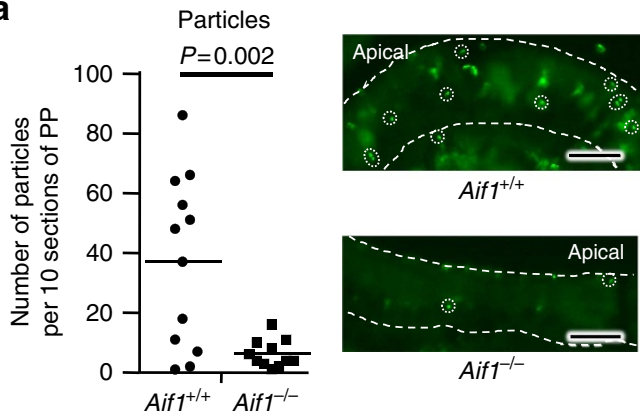

Aif1 $^{-1-}$

b
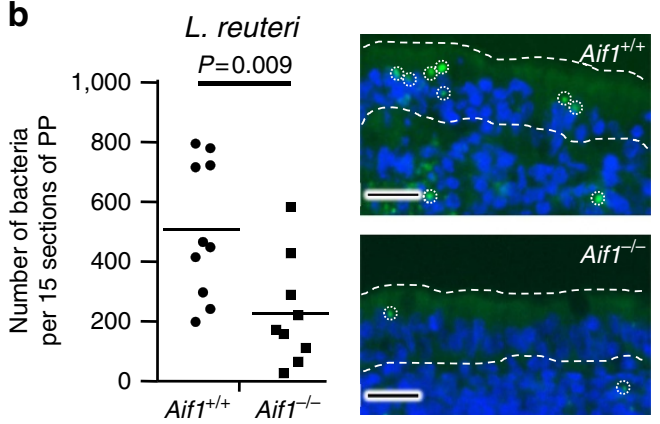

Figure 4 | Influence of Aif1 deficiency on transcytosis function of $\mathbf{M}$ cells. Evaluation of particle (a) and Lactobacillus reuteri (b) sampling by $M$ cells. Mice fasted for $24 \mathrm{~h}$ were given fluorescence-conjugated $200 \mathrm{~nm}$ particles $\left(1 \times 10^{11}\right.$ beads $)$ or FITC-labelled bacteria $\left(5 \times 10^{9} \mathrm{CFUs}\right)$ orally. Two hours later, PPs were collected from the duodenum, fixed in $4 \%$

paraformaldehyde, and cut into 10 or 15 consecutive sections. The numbers of particles or labelled bacteria that had entered each PP were then counted under a fluorescence microscope. Each dot represents an individual PP from three independent experiments (For particles: Aif1 ${ }^{+/+}, n=12 ;$ Aif $^{-/-}$, $n=11$. For L. reuteri: Aif1 ${ }^{+/+}, n=10 ;$ Aif1 ${ }^{-/-}, n=9$ ). Horizontal bars indicate each mean. $P$ values were determined with Student's $t$-test. Right panels are representative images of each section. Dotted lines indicate FAE regions and circles indicate each particle or bacterium. Scale bar, $20 \mu \mathrm{m}$.

lymphocytes, contributed to the uptake of particles and $Y$. enterocolitica. We used BM chimeric mice prepared by transplanting Aif $1^{-1-} \mathrm{BM}$ cells into congenic wild-type (Ly5.1) mice (Aif1 ${ }^{-/-} \mathrm{BM} \rightarrow$ Ly5.1) or vice versa (Ly5.1 BM $\rightarrow$ Aif $1^{-/-}$). Both types of chimeric mice were orally given the microparticles ( $200 \mathrm{~nm}$ in diameter) or Y. enterocolitica 8 weeks after BM transfer. Uptake of either particles or $Y$. enterocolitica was not influenced by Aif1-deficient BM transfer in Ly5.1 mice (Fig. 6a,b). Transfer of wild-type $\mathrm{BM}$ to Aif1 $1^{-/-}$mice did not improve the significantly reduced particle or $Y$. enterocolitica uptake in these mice compared with that in the Aif1-/- BM $\rightarrow$ Ly5.1 mice. These results suggest that Aif1 expression in $M$ cells, but not in haematopoietic cells, contributes to the uptake of particles and Y. enterocolitica.

Aif1-deficient mice showed impaired uptake of the major enteric commensal bacterium, L. reuteri (Fig. $4 \mathrm{~b}$ ). Given that M-cell-intrinsic Aif1 is important for the uptake of substantial numbers of commensal bacteria, it is possible that secretory $\operatorname{IgA}(\mathrm{SIgA})$ production is also diminished in Ly5.1 $\mathrm{BM} \rightarrow$ Aif1 $^{-/}$chimeric mice, because IEC-specific RANK-deficient mice, which lack PP M cells, have less faecal SIgA than do control mice ${ }^{30}$. Enzyme-linked immunosorbent assay (ELISA) examination of the amounts of faecal total IgA in both types of Aifl chimeric mice revealed
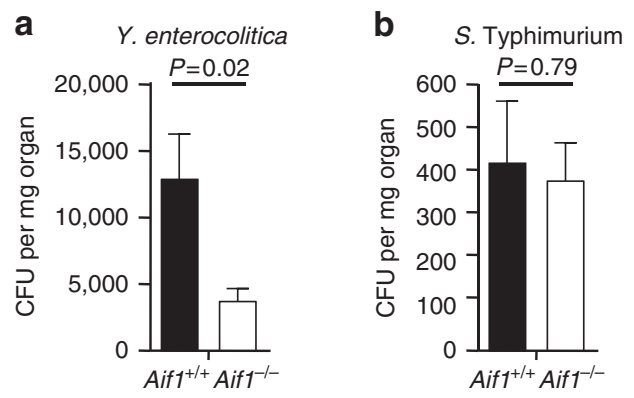

C
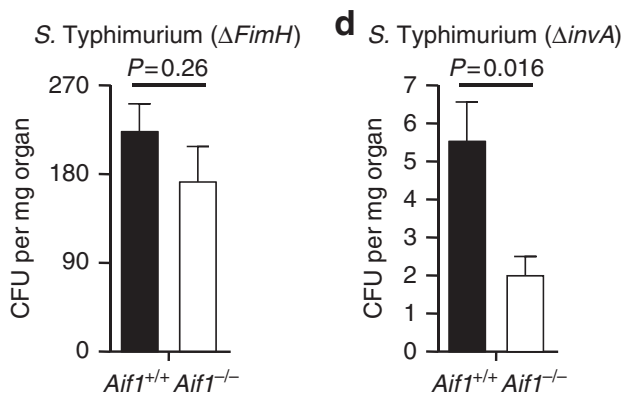

Figure 5 | Impaired translocation of pathogenic bacteria in Aif1 ${ }^{-/-}$ mice. Evaluation of transcytosis of $Y$. enterocolitica $\left(\mathbf{a} ; 1 \times 10^{9} \mathrm{CFUs}\right.$ given orally) and indicated strains of $S$. Typhimurium (b-d; $1 \times 10^{8}$ CFUs given orally). Twenty-four hours after administration of the bacteria, PPs were collected from the ileum, homogenized and diluted. Each diluted sample was cultured on the appropriate plates. Colonies were counted and the values normalized against the weight of the PPs. Data are shown as means \pm s.e.m. from two independent experiments ( $n=18$ per genotype for $Y$. enterocolitica; $n=10$ to 20 per genotype for $S$. Typhimurium). $P$ values were determined with Student's t-test.
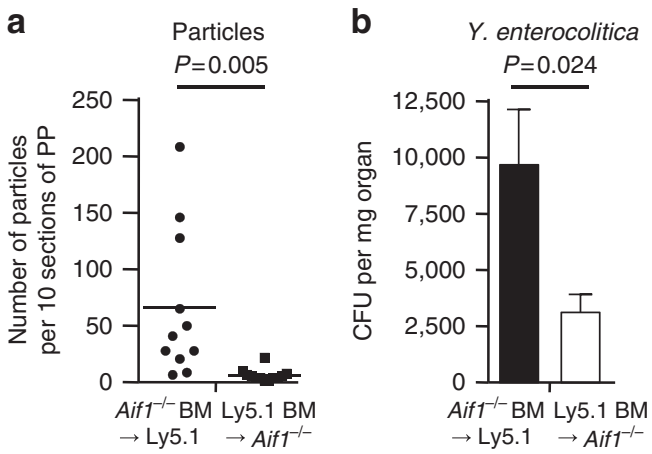

Figure 6 | Importance of M-cell-intrinsic Aif1 for uptake of particles and Y. enterocolitica. Evaluation of $200 \mathrm{~nm}$ bead uptake (a) and transcytosis of $Y$. enterocolitica (b) in bone-marrow (BM) chimeric mice to which we transferred Aif1 ${ }^{-/}-\mathrm{BM}$ cells (Aif1-/- BM $\rightarrow$ Ly5.1) or vice versa (Ly5.1 BM $\rightarrow$ Aif $^{-/-}$). Both experiments used the same protocols as described in Fig. 4a (particles) and Fig. 5a (Y. enterocolitica; for particles: $n=12$ per group. For Y. enterocolitica: Aif1 ${ }^{-/-}$BM $\rightarrow$ Ly5.1, $n=5$; Ly5.1 BM $\rightarrow$ Aif1 $\left.{ }^{-} /-, n=6\right)$. $P$ values were determined with Student's t-test.

no significant difference between the two groups (Fig. 7a), suggesting that entry of commensal bacteria was not totally impaired in Ly5.1 $\mathrm{BM} \rightarrow$ Aif1 $1^{-/-}$chimeric mice. Interestingly, however, the population of IgA-coated faecal bacteria was significantly reduced in Ly5.1 BM $\rightarrow$ Aif $1^{-/-}$ chimeric mice compared with that in Aif1 $^{-/-} \mathrm{BM} \rightarrow$ Ly5.1 mice (Fig. 7b,c). 
a

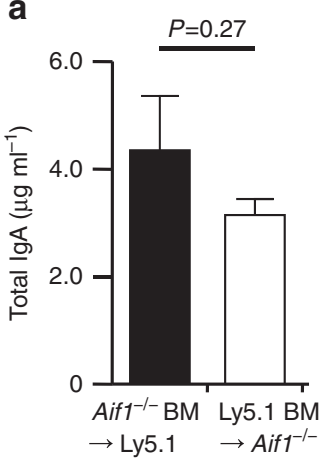

d

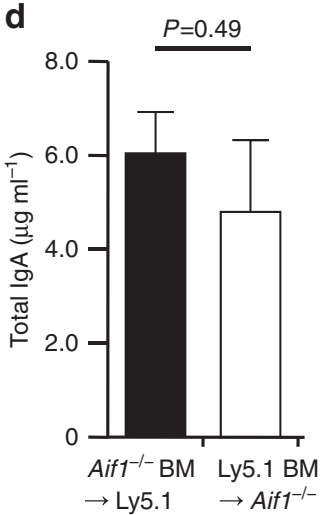

b

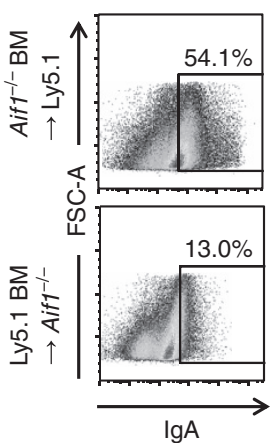

e

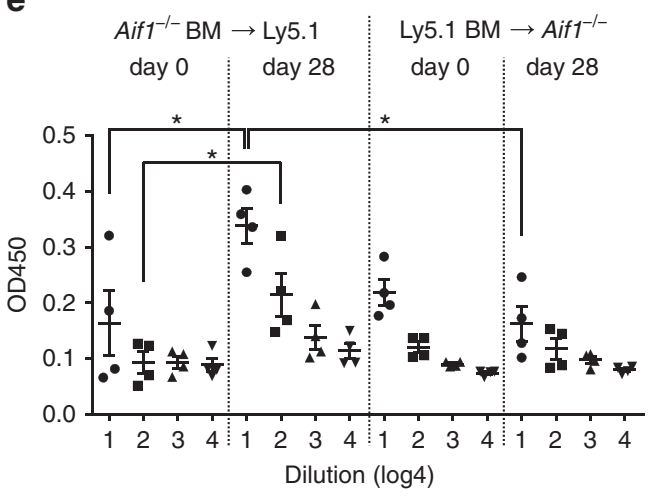

Figure 7 | Importance of Aif1 for initiation of the mucosal IgA immune response. (a) Quantification of total faecal IgA. Faeces were prepared from the chimeric mice indicated. Concentrations of total IgA were determined by ELISA. Data are means \pm s.e.m. from two independent experiments $(n=8$ per group). $P$ value was determined with Student's $t$-test. (b) Representative plots of bacterial flow cytometry. Faeces were collected from the chimeric mice indicated and suspended in PBS. Bacteria recognized by IgA were detected and their percentages determined. (c) The percentages determined in $\mathbf{b}$ are shown as means \pm s.e.m. from two independent experiments $(n=12$ per group). $P$ value was determined with Student's $t$-test. (d) Quantification of total IgA after long-term infection with $Y$. enterocolitica. Faeces were prepared from the indicated chimeric mice on day 28 after infection. Concentrations of total $\lg A$ were determined by ELISA. Data are means \pm s.e.m. from two independent experiments ( $n=6$ per group). $P$ value was determined with Student's t-test. (e) Comparison of anti-Y. enterocolitica-specific lgA production after long-term infection. Serial diluted faecal extracts prepared from $Y$. enterocolitica-infected mice were subjected to ELISA by using $Y$. enterocolitica-coated plates. Data are means \pm s.e. $m$. from one experiment representative of two independent experiments ( $n=4$ per group). ${ }^{\star} P<0.05$, determined with Student's $t$-test.

Next, we measured anti-Y. enterocolitica-specific antibody in a model of long-term infection. Y. enterocolitica at $1 \times 10^{8}$ CFUs was given orally to the chimeric mice once a week to analyse total and antigen-specific IgA antibody induction. After four inoculations of $Y$. enterocolitica, Ly5.1 recipient mice, which possessed wild-type $M$ cells and Aif1-deficient haematopoietic cells, produced large amounts of total and anti-Y. enterocolitica faecal IgA antibodies (Fig. 7d,e). Although Aif1 ${ }^{-/-}$recipient mice, which possessed Aif1-deficient $M$ cells and wild-type haematopoietic cells, had comparable levels of total IgA in their faeces after infection as before infection (Fig. 7a,d), no induction of anti-Y. enterocolitica-specific IgA production was observed in these mice. These data strongly suggest that M-cell-intrinsic Aif1 expression is important to the translocation of $Y$. enterocolitica, and that this translocation was followed by the induction of a mucosal IgA immune response to this bacterium.

Aif1-dependent activation of $\beta 1$ integrin on M-cell surface. Invasin, which is one of the outer proteins of $Y$. enterocolitica, is a specific ligand molecule that binds to $\beta 1$ integrin on the apical surfaces of $\mathrm{M}$ cells to facilitate effective entry of $Y$. enterocolitica into $\mathrm{PP}^{31-34}$. We hypothesized that apical surface expression or activation, or both, of $\beta 1$ integrin in M cells would be regulated by Aif1 and would thus be diminished in Aif1-deficient mice. However, the level of $\beta 1$ integrin mRNA (Itgb1) expression did not differ significantly between FAEs prepared from control and Aif1 ${ }^{-1-}$ mice (Fig. 8a). Next, we examined the surface expression of total and activated $\beta 1$ integrin on the apical side of $M$ cells by using whole-mount staining without cell permeabilization. We examined the apical side because non-M-cell enterocytes also express $\beta 1$-integrin on the lateral membranes, but not on the apical surfaces ${ }^{34}$. Total $\beta 1$ integrin expression was detected on the apical surfaces of GP2-positive M cells in control and Aif1 ${ }^{-/-}$mice (Fig. 8b), whereas active $\beta 1$ integrin expression on the apical surfaces of $\mathrm{M}$ cells was seen in control mice but not in Aif1-deficient mice (Fig. 8c). These data suggested that Aif1 was important for the activation of $\beta 1$ integrin on the apical surfaces of $M$ cells contributing to the translocation of $Y$. enterocolitica via invasin.

\section{Discussion}

Here we identified Aif 1 as a novel molecule specifically expressed in $M$ cells among IECs, and we newly generated Aif1-deficient mice for in vivo elucidation of the role of Aifl in M-cell development and function. We demonstrated that Aif1 is important for the M-cell uptake function of extraneous 

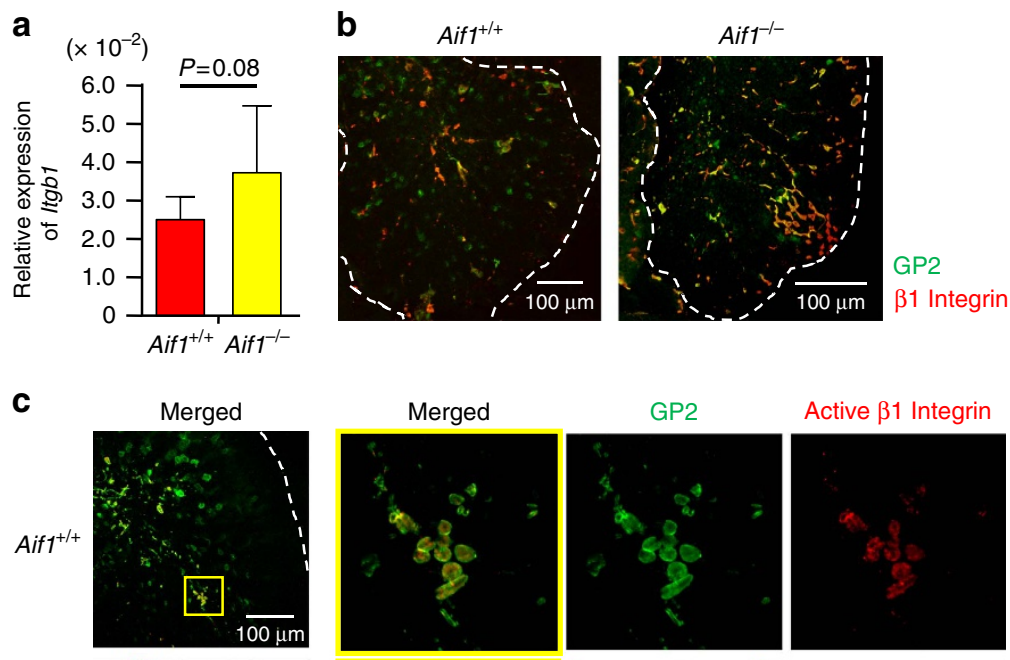

Active $\beta 1$ Integrin
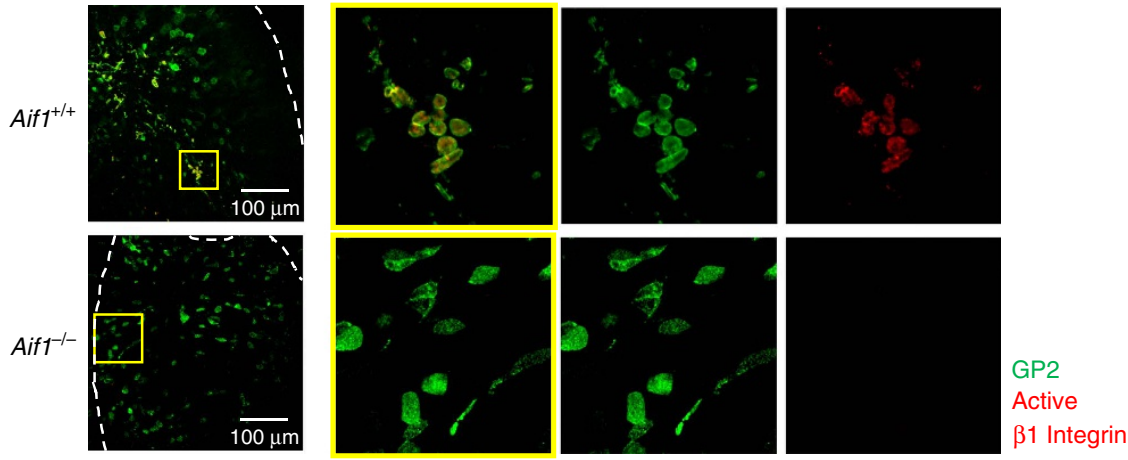

Figure 8 | Aif1-dependent activation of $\boldsymbol{\beta} 1$ integrin. (a) Relative gene expression of $\beta 1$ integrin in FAE from wild-type and Aif1 ${ }^{-/-}$mice. Data are means \pm s.e.m. of one experiment representative of two independent experiments ( $n=4$ per genotype). $P$ value was determined with Student's $t$-test. $(\mathbf{b}, \mathbf{c})$ Whole-mount staining of PPs prepared from Aif1 ${ }^{+/+}$and Aif1-/- mice with anti-total $\beta 1$ integrin antibody (b, red) or anti-activated- $\beta 1$ integrin antibody (c, red), together with anti-GP2 antibody (b,c, green). Each yellow box region in c (left panels) was magnified to produce the image in $\mathbf{c}$ (right panels). Dotted lines indicate the periphery of the FAE region. Data are representative of three independent experiments.

substances such as microparticles and certain commensal bacteria, including $L$. reuteri, but that it is not involved in the structural development of $\mathrm{M}$ cells. Furthermore, Aif1 is involved in the activation of $\beta 1$ integrin, which is a potential receptor for the invasin of $Y$. enterocolitica, at the apical surface of $M$ cells for the effective entry of this pathogenic bacterium into $\mathrm{PPs}$ and the subsequent induction of antigen-specific IgA immune responses.

Because Aif1 is also strongly expressed in the testis, it has been hypothesized that Aif1 has a role in spermatogenesis ${ }^{19,35}$. However, our strain, and another strain, of Aif1-deficient male mice showed normal fertility (data not shown and ref. 36). Aif1 expression has also been found in another epithelial cell, namely the glomerular visceral epithelial cell, or podocyte, which partially covers the outside of the glomerular basement membrane and acts as a final barrier to protein loss in the kidney ${ }^{37}$. The function of Aif1 in podocytes is unknown; however, it very likely has a role related to the transport of materials, because it is expressed in epithelial cells specialized for substance transfer or filtration, such as the $M$ cells in IECs.

Aif1 expression in $M$ cells was totally dependent on Spi-B, which is a master regulator of $\mathrm{M}$-cell development ${ }^{16-18}$. Interestingly, however, gene expression and protein production of Aif1 in the haematopoietic cells in PPs were not affected by Spi-B deficiency (Fig. 1a; Supplementary Fig. 4). Recently, it has been reported that expression of Aif1 in microglia is dependent on interferon regulatory factor 8 (IRF8) ${ }^{38,39}$. In addition, expression of Aif1 in microglial cells and IFN $\gamma$-stimulated macrophages requires the transcription factor PU.1 (refs 40,41). In fact, in the predicted promoter region of Aif1 there are several consensus binding sites for transcription factors, including those of the Ets, IRF and STAT families ${ }^{40}$. Because PU.1 belongs to the Ets family and, among the members of that family, is closest to Spi-B, it is possible that Aif1 is a direct target of Spi-B in $\mathrm{M}$ cells. Another explanation for the regulation of Aif1 is that another transcription factor-perhaps PU.1 or IRF8-is upregulated or activated, or both, in $\mathrm{M}$ cells to trigger the transcription of Aif1.

Numerous in vitro experiments have indicated that Aif1 is involved in actin polymerization in inflammation ${ }^{42,43}$. In fact, in a collagen-induced arthritis model, Aif1-deficient mice showed attenuated signs of arthritis ${ }^{36}$. There have been also many reports that Aif1 is involved in actin remodelling, ruffling and bundling as part of the phagocytic function of macrophages and microglial cells ${ }^{20-22,29}$ and as part of the migration of vascular smooth muscle cells ${ }^{23}$. Therefore, we hypothesized that Aif1 deficiency would affect the ultrastructure of $\mathrm{M}$ cells. However, a sunken structure, which is one of the features of the apical surfaces of $M$ cells $^{7,12}$, was easily and consistently detected in our Aif1-deficient mice (Fig. 3a,b). Transmission electron microscopic analysis also revealed that Aif1-deficient mice possessed cells with M-cell morphology in their FAE, which had irregular microvilli and endosomes on the apical side and a pocket structure on the basolateral side (Fig. 3c,d). Furthermore, whole-mount staining showed that the numbers of GP2-positive cells found in FAE were almost the same in wild-type and Aif1-deficient mice (Fig. 2b,c). Interestingly, although Aif1 is presumably a cytoplasmic molecule, $Z$-stacked transverse images from whole-mount staining showed that localisation of Aif1 was abundant on the apical side, but not the basolateral side, of $M$ cells (Supplementary Fig. 3). These data suggest that Aif1 is not involved in actin remodelling on the basal side, nor in the fundamental structural morphology or maturation of $\mathrm{M}$ cells.

Our study also demonstrated that uptake of particles and $L$. reuteri by $M$ cells was significantly less in Aif1 $1^{-/-}$mice than in wild-type mice (Fig. 4a,b). These results suggest that Aif1 plays a critical role in M-cell-mediated antigen uptake from 
the intestinal lumen-a unique characteristic of these cells. It remains unknown precisely what stimuli activate Aifl; however, it is interesting to postulate that Aif1 acts when transcytosis has just occurred at the apical surface of the $\mathrm{M}$ cell, because Aif1 deficiency seemed not to affect cell-structural morphology. In relation to this issue, although the percentage of SIgA-coated faecal bacteria was significantly lower in Ly5.1 $\mathrm{BM} \rightarrow$ Aif $^{-/-}$chimeric mice than in Aif $1^{-/-}$ $\mathrm{BM} \rightarrow$ Ly5.1 mice, certain bacteria were still recognized by SIgA in the Ly5.1 $\mathrm{BM} \rightarrow A$ if $1^{-/-}$mice (Fig. $7 \mathrm{~b}, \mathrm{c}$ ), because no IgA-coated bacteria were observed in faeces prepared from Rag1-deficient mice, which lack $\mathrm{T}$ and $\mathrm{B}$ cells. Taken together with the evidence that total $\operatorname{IgA}$ production levels were comparable in the above mentioned two types of chimeric mice (Fig. 7a), these results suggest that Aif1-independent uptake of some kinds of commensal bacteria occurs continuously at M cells. This might also explain why endosomes were still observed in Aif1-deficient $\mathrm{M}$ cells (Fig. 3d). One possible mechanism of Aif1-independent uptake is IgA-mediated transcytosis by Dectin-1 because it has recently been reported that Dectin-1 acts on IgA-mediated transcytosis in M-like cells derived from Caco-2 cells, a human colonic cancer cell line, and mouse $\mathrm{M}$ cells ${ }^{44}$. However, we considered that Dectin-1 is not contributed on SIgA-mediate antigen uptake on $M$ cells because the Dectin-1 mRNA level in the FAE of mouse PPs was very low (data not shown). In addition, we could not detect Dectin-1-positive cells in mouse PP FAE by whole-mount staining using anti-mouse Dectin-1 mAb (clone: RH1; Supplementary Fig. 6), indicating that Dectin-1 is not expressed on the apical side of $\mathrm{M}$ cells. Furthermore, Dectin-1 has never been identified from several gene-expression and profiling data sets as an FAE- or M-cell-specific gene.

In addition to the defects in uptake of particles and some kinds of commensal bacteria in Aif1-deficient mice, invasion of $\mathrm{M}$ cells by $Y$. enterocolitica was also significantly decreased in these mice, whereas uptake of $S$. Typhimurium was normal (Fig. 5a,b). Upon the entry of pathogenic bacteria into host epithelial cells, reorganisation of the actin cytoskeleton is induced by 'zipper' or 'trigger' mechanisms ${ }^{27,28}$. The zipper mechanism, which is induced upon invasion by Yersinia, Listeria and Neisseria spp., is initiated by bacterial ligand and host cellular receptor interaction. On the other hand, the trigger model is observed upon entry of Salmonella and Shigella spp., with the induction of membrane ruffling. Given that Aif1 is associated with the activation of $\beta 1$ integrin, but not with the expression of $\mathrm{GP} 2$, in $\mathrm{M}$ cells (Fig. 8b,c and 2b), it is possible that actin rearrangement at the apical side of $M$ cells is not induced by $Y$. enterocolitica in Aif1-deficient mice, because $\beta 1$ integrin acts as a receptor for the invasin molecules of Yersinia spp. ${ }^{31-34}$. Consistently, uptake of $\Delta i n v A S$. Typhimurium, which did not induce membrane ruffling, was significantly lower in Aif1-deficient mice than in control mice (Fig. 5d).

Our data strongly indicate that M-cell-intrinsic Aif1 is important for not only uptake of particles, some kinds of commensal bacteria, and $Y$. enterocolitica, but also for the subsequent activation of mucosal IgA immune responses. Because CD11c-positive cells in PPs and the lamina propria also expressed Aif1 (Supplementary Fig. 1), Aif1 might contribute to antigen presentation by antigen-presenting cells or to antigen sampling by $\mathrm{CD} 11 \mathrm{c}^{+} \mathrm{CX}_{3} \mathrm{CR} 1^{+}$dendritic cells, which are found in the sub-epithelial dome region of PPs and can directly sample bacteria from the intestinal lumen ${ }^{45,46}$. However, this possibility was contradicted, because the wild-type mice to which we adoptively transferred Aif1 $1^{-/-}$haematopoietic cells (Aif1 ${ }^{-/}-\mathrm{BM} \rightarrow$ Ly5.1: mice harbouring normal $\mathrm{M}$ cells and Aif $1^{-/-}$antigen-presenting cells) showed induction of antigen-specific SIgA production, whereas this was severely defective in our other chimeric mice (Ly5.1 $\mathrm{BM} \rightarrow$ Aif $1^{-/-}$: mice harbouring Aif1 $1^{-/-} \mathrm{M}$ cells and normal antigen-presenting cells) after Y. enterocolitica infection (Fig. 7e).

Small GTPases contribute to the activation of integrins via $\mathrm{Ca}^{2+}$-binding molecules such as calmodulin ${ }^{47}$. Because Aif1 has $\mathrm{Ca}^{2+}$-binding EF hand motifs, and because we previously reported that Rac2 is specifically expressed in $\mathrm{M}$ cells among IEC lineages ${ }^{13}$, it is possible that Rac2 mediates Aif1-dependent $\beta 1$ integrin activation in $M$ cells. In fact, Aif1-mediated activation of Rac2 has been shown to be important for the activation of vascular smooth muscle cells ${ }^{48}$. Taken together with the data shown in this study, it can be suggested that Aifl expression in $M$ cells contributes to the activation of Rac2, leading to (1) activation of $\beta 1$ integrin, which contributes to the translocation of $Y$. enterocolitica, and (2) actin remodelling, which contributes to the uptake of particle antigens and enteric commensal bacteria by $M$ cells, though future studies are required to elucidate further the mechanisms of how Aif1 controls actin remodelling and the activation of $\beta 1$ integrin in vivo.

\section{Methods}

Generation of Aif1-deficient mice. A mouse Aif1 genome clone (GenBank Accession \# AB036423) was isolated by using a mouse Aif1 cDNA probe to screen a 129 SVJ mouse genome cosmid library (Stratagene). The targeting vector was constructed on pBluescriptIISK(-) (Stratagene) by inserting a diphtheria toxin A (DT-A) expression cassette, a KpnI-HindIII fragment containing Aif1 exons 1 and 2 and part of exon 3, a neomycin resistance cassette, a HindIII-NotI fragment corresponding to the $3^{\prime}$-downstream region of Aif1, and another DT-A cassette (Supplementary Fig. 5a). The HindIII site of the $3^{\prime}$-fragment was destroyed by ligation with the neomycin cassette. The vector was linearized by digestion with XhoI and electroporated into embryonic stem cells. Embryonic stem cell clones selected by G418 resistance were screened for homologous recombination by Southern blot analysis (data not shown). These clones were individually microinjected into blastocysts derived from C57BL/6 mice and transferred to pseudopregnant females. A chimeric female mouse was mated with a 129 male mouse; their offspring, which were judged as Aif1 heterologously deleted $\left(A i f 1^{+/-}\right)$, were then backcrossed for more than eight generations with C57BL/6J mice. Heterozygous mice were crossed to obtain Aif1 $1^{+1+}$ and Aif $1^{-/-}$mice. Littermates or age- and gender-matched Aif $1^{+/+}$and Aif $1^{-/}$ mice were used for all subsequent experiments in this study. Aif1 genotyping was performed by PCR method using the sets of primers: for wild-type allele, $5^{\prime}$-GGCTTCAAGTTTGGACGGCAGATCCTC- ${ }^{\prime}$ and $5^{\prime}$-CATGAGCCAAAGC AGGGATTTGCAGGG- ${ }^{\prime}$; and for mutated allele, $5^{\prime}$-ATGAGCCAAAGCAG GGATTTG- $3^{\prime}$ and $5^{\prime}$-CGACCTGCAGCCAATATGGG- $3^{\prime}$.

Mice. Spib ${ }^{-/-}$mice (C57BL/6 background) were kindly provided by Dr M. Celeste Simon (University of Pennsylvania). CD45.1 congenic mice on the C57BL/6 background (Ly5.1) and Rag1 ${ }^{-1}$ mice on the C57BL/6 background were purchased from Jackson Laboratory. C57BL/6J mice were obtained from CLEA Japan. Until use, all mice were maintained under specific-pathogen-free conditions in horizontal laminar flow cabinets in the experimental animal facility of the Institute of Medical Science, The University of Tokyo. Six to 12 weeks old mice of both genders were used in this study. All animal experiments were conducted with the approval of the Animal Research Committee of the Institute of Medical Science, The University of Tokyo.

Real-time PCR. To isolate and prepare FAE from PPs, dissected PPs were washed and then incubated in PBS containing $30 \mathrm{mM}$ EDTA and $1 \mathrm{mM}$ DTT for 30 min on ice. The FAE was peeled off by manipulation with fine needles under a stereoscopic microscope. Total RNA was prepared from the cells by using an RNeasy Kit (Qiagen). Complementary DNA was reverse transcribed by using SuperScript VILO master mix (Invitrogen), and then used as a template for real-time PCR with a set of specific primers and a LightCycler 480 SYBR Green I Master (Roche) on a LightCycler 480 System II (Roche). Expression levels of target genes were normalized against that of glyceraldehyde 3-phosphate dehydrogenase (Gapdh). The sets of primers used were as follows: Gapdh (forward) $5^{\prime}$-TGTCCGTCGTGGATCTGAC-3 ${ }^{\prime}$ and (reverse) $5^{\prime}$-CCTGCTTCACCAC CTTCTTG- $3^{\prime}$; Aif1 (forward) $5^{\prime}$-GGATTTGCAGGGAGGAAAA-3' and (reverse) $5^{\prime}$-TGGGATCATCGAGGAATTG-3'; Gp2 (forward) $5^{\prime}$-ATACTGC ACAGACCCCTCCA- $3^{\prime}$ and (reverse) $5^{\prime}$-GCAAACTCTGAGAGTCAGAA ACATG-3'; Spib (forward) $5^{\prime}$-GCCCACACTTAAGCTGTTTGTA-3' and (reverse) $5^{\prime}$-CTGTCCAGCCCCATGTAGAG-3'; and Itgbl (forward) $5^{\prime}$-ctgcttcta aaattgagatcagga- $3^{\prime}$ and (reverse) $5^{\prime}$-tccataggtagtagagatcaataggg- $3^{\prime}$ 
Electron microscopic analysis. PPs dissected from the ileum were fixed at room temperature for $2 \mathrm{~h}$ in a solution containing $2.5 \%$ glutaraldehyde, $2 \%$ paraformaldehyde and $0.1 \mathrm{M}$ phosphate buffer ( $\mathrm{pH}$ 7.4). After being washed with $3 \%$ sucrose three times on ice, the samples were fixed in $1 \%$ osmium tetraoxide on ice for $1 \mathrm{~h}$ and dehydrated sequentially with $30,50,70$ and $90 \%$ ethanol for $15 \mathrm{~min}$ each. For scanning electron microscopy, dehydrated samples were freeze-embedded in $t$-butyl alcohol and freeze dried, and then coated with osmium. Each sample was then observed under a scanning electron microscope (S-4200; Hitachi). For transmission electron microscopy, dehydrated samples were treated with propylene oxide twice for $5 \mathrm{~min}$, and then embedded in Epon812 resin mixture (TAAB Laboratories). Ultra-thin sections $(70 \mathrm{~nm})$ stained by $2 \%$ uranyl acetate and Reynolds lead solution were examined under a Hitachi H-7500 electron microscopy.

Whole-mount staining. PPs were excised from the duodenum and ileum and then washed with PBS. The PPs were then fixed, permeabilized, and blocked with a BD Cytofix/Cytoperm kit (BD Biosciences) or just fixed by $4 \%$ paraformaldehyde and blocked with $0.2 \%$ BSA/PBS. Permeabilized tissues were stained with rat antimouse GP2 antibody (MBL) and rabbit anti-mouse Aif1 antibody (Wako), followed by the appropriate secondary antibodies conjugated with Alexa Fluor 488 or Cy3 (Jackson ImmunoResearch). After being washed with PBS, the specimens were stained with Alexa Fluor 633-conjugated Phalloidin (Invitrogen). Unpermeabilized tissues were stained with rat anti-mouse integrin beta 1 (clone 265917, R\&D) or rat anti-mouse active integrin beta 1 (clone 9EG7, BD Biosciences), followed by Cy3-conjugated secondary anti-rat Ig antibody. After being washed, samples were then stained with Alexa Fluor 488-conjugated anti-mouse GP2 antibody (MBL). Samples were mounted and analysed under a confocal laser-scanning microscope (DM IRE2/TCS SP2; Leica or FV1200; Olympus).

Quantification of antigen uptake by $\mathbf{M}$ cells. About $1 \times 10^{11}$ particles $(200 \mathrm{~nm}$ in diameter; Yellow Green Microspheres, Polysciences) or FITC-labelled L. reuteri $\left(5 \times 10^{9}\right.$ CFUs) were given orally to mice that had been fasted for $24 \mathrm{~h}$. Two hours later, two PPs were dissected from the duodenum of each mouse and embedded in OCT compound. After we had made 10 or 15 successive frozen sections, the particles or bacteria found inside the PPs were observed and counted under a fluorescence microscope (Keyence).

Bacteria. Lactobacillus reuteri (ATCC 23272) obtained from RIKEN BioResource Center were grown overnight at $37^{\circ} \mathrm{C}$ under anaerobic conditions while left standing in MRS broth (BD Biosciences). For FITC labelling, $5 \times 10^{9}$ L. reuteri were incubated with $5 \mathrm{mg} \mathrm{ml}^{-1}$ Fluorescein isothiocyanate isomer I-Celite (Sigma) at $37^{\circ} \mathrm{C}$ for $60 \mathrm{~min}$ with shaking. Nalidixic acid-resistant $S$. Typhimurium $(\text { strain } \chi 3306)^{49}$ and invA-deletion mutants of $\chi 3306$ (strain UF102) ${ }^{50}$, which possess a kanamycin resistance gene, were kindly provided by Dr Hidenori Matsui of Kitasato University. To generate FimH-deletion mutants of $S$. Typhimurium, pKD46 plasmid encoding Red recombinase ${ }^{51}$ was introduced into $\chi^{3306 .} \chi 3306$ carrying pKD46 were then transformed with a PCR fragment containing the kanamycin resistance cassette and regions homologous to the FimH gene; the fragment was prepared with $\mathrm{pKD} 4$ plasmid as a template and the primer set of 5'-CAACCTGTATCCGTCCGGCGTCATAAAAGGAAAAATAGAGGTGTA GGCTGGAGCTGCTTC- ${ }^{\prime}$ and $5^{\prime}$-CGATAGCGATGAAAACGCGCC GAAGGATCATTATGCCTCCATGGGAATTAGCCATGGTCC-3' . After culture of the above transformants in LB broth containing $\mathrm{L}$-arabinose and kanamycin, ampicillin-sensitive clones that had lost the pKD46 were selected and then checked for FimH deletion by PCR. All S. Typhimurium strains were grown overnight at $37^{\circ} \mathrm{C}$ in LB broth containing nalidixic acid (and kanamycin for strain UF102 and $\Delta$ FimH). Y. enterocolitica (ATCC 27729) was grown overnight at $26^{\circ} \mathrm{C}$ in tryptic soy broth. Following overnight incubation, a culture of $S$. Typhimurium and one of Y. enterocolitica were prepared such that the $\mathrm{OD}_{600}$ reached 0.1 to 0.2 ; they were placed into fresh growth medium and further incubated for $2 \mathrm{~h}$ at the appropriate temperatures for each, as above.

Bacterial administration assay. Aif $1^{+/+}$and $A i f 1^{-/-}$mice were each given $100 \mu \mathrm{l}$ of $1 \times 10^{9} \mathrm{CFU}$ per ml $S$. Typhimurium or $1 \times 10^{10} \mathrm{CFU}$ per $\mathrm{ml}$ Y. enterocolitica by mouth. After $24 \mathrm{~h}$, PPs were dissected from the ileum, washed with PBS, and then incubated in $100 \mu \mathrm{g} \mathrm{ml}^{-1}$ gentamycin solution at room temperature for $30 \mathrm{~min}$. After being washed with PBS, PPs were weighed and homogenized. The diluted homogenate samples were plated on LB agar plates containing $25 \mathrm{\mu g} \mathrm{ml}^{-1}$ nalidixic acid (for $S$. Typhimurium $\chi^{3306}$ ) and $50 \mu \mathrm{g} \mathrm{ml}^{-1}$ kanamycin (for UF102 and $\Delta$ FimH), or on Yersinia-selective agar plates (Kanto Chemical Co.; for Y. enterocolitica). Data were excluded if no colonies were observed even in the highest amounts plating plate.

BM transfer. BM cells were prepared from Aif1 $1^{-/}$mice (CD45.2) and congenic Ly5.1 mice (CD45.1). The cells were transferred to Ly5.1 and Aif $1^{-/-}$mice, respectively, the haematopoietic cells of which had been disrupted by radiation $(9.5 \mathrm{~Gy})$. All mice were used for experiments 8 weeks after BM transfer. To confirm chimerism, flow cytometric analysis was performed to splenocytes prepared from all mice used in the experiments with antibodies to CD45.1 and CD45.2 (clones A40 and 104, respectively; BioLegend). At least 95\% of the haematopoietic cells were replaced by donor cells (data not shown).

Flow cytometric analysis of IgA-coated bacteria in faeces. Faeces were collected and weighed, and then suspended in PBS to $100 \mathrm{mg} \mathrm{ml}^{-1}$ by vigorous vortexing at $4{ }^{\circ} \mathrm{C}$ for $30 \mathrm{~min}$. Each $500-\mu \mathrm{l}$ suspension was centrifuged at $4{ }^{\circ} \mathrm{C}$, 13,000 r.p.m. for $10 \mathrm{~min}$. The pellet was then suspended in $1 \mathrm{ml} 4 \%$ paraformaldehyde and incubated at $4{ }^{\circ} \mathrm{C}$ overnight with rotation. Each suspension was centrifuged at $4^{\circ} \mathrm{C}, 1,000$ r.p.m. for $2 \mathrm{~min}$, and then $5 \mu \mathrm{l}$ of the supernatant was washed twice with FACS buffer (2\% FBS in PBS). Pellets were stained with FACS buffer containing $1 \mathrm{mg} \mathrm{ml}^{-1}$ rat serum (Jackson ImmunoResearch) and $1 \mu \mathrm{g} \mathrm{ml}^{-1}$ PE-conjugated anti-mouse IgA (clone $\mathrm{mA}-6 \mathrm{E} 1$, eBioscience) at $4{ }^{\circ} \mathrm{C}$ for $20 \mathrm{~min}$, and were then analysed on NovoCyte Flow Cytometer Systems (ACEA). IgA-positive gate was determined by using isotype control antibody (Rat IgG1 $\kappa$, clone: RTK2071, BioLegend; Supplementary Fig. 7).

Detection of $\mathbf{Y}$. enterocolitica-specific antibody response. $Y$. enterocolitica $\left(1 \times 10^{8} \mathrm{CFUs}\right)$ was given orally to BM-transferred chimeric mice once a week. Four weeks after the first inoculation, faeces were collected from the mice. Supernatants were prepared from $100 \mathrm{mg} \mathrm{ml}^{-1}$ diluted faeces, which were vigorously shaken and centrifuged at $4^{\circ} \mathrm{C}$. MaxiSorp plates (Thermo) were coated with $100 \mu \mathrm{l}$ of $1.0 \mathrm{mg} \mathrm{ml}^{-1}$ lyophilized Y. enterocolitica. The plates were washed four times with $0.05 \%$ Tween 20 in PBS (PBS-T) and then blocked with $1 \%$ BSA in PBS-T for $2 \mathrm{~h}$ at room temperature; the diluted samples were incubated for $2 \mathrm{~h}$ at room temperature. After the samples had been washed with PBS-T, they were incubated with HRP-conjugated anti-mouse IgA antibodies (Southern Biotech) for $2 \mathrm{~h}$ at room temperature. After washing of the samples with PBS-T again, a 3,3',5,5' -tetramethylbenzidine Microwell Peroxidase Substrate system (Kirkegaard \& Perry Laboratories) was used for detection according to the manufacturer's protocol. Absorbance was read at a wavelength of $450 \mathrm{~nm}$ with a SmartSpec Plus Spectrophotometer and an iMark Microplate Reader (Bio-Rad).

Statistical analysis. Results were compared by using an unpaired two-tailed Student's $t$-test. Statistical significance was established at $P<0.05$. All statistical analyses were conducted by using GraphPad Prism 6. No statistical methods were used to determine sample size. These experiments were not randomized and the investigators were not blinded to allocation during experiments and outcome assessment.

Data availability. The data that support the findings of this study are available from the corresponding author upon request.

\section{References}

1. Abreu, M. T., Fukata, M. \& Arditi, M. TLR signaling in the gut in health and disease. J. Immunol. 174, 4453-4460 (2005).

2. Fagarasan, S. \& Honjo, T. Regulation of IgA synthesis at mucosal surfaces. Curr. Opin. Immunol. 16, 277-283 (2004).

3. Madara, J. L., Nash, S., Moore, R. \& Atisook, K. Structure and function of the intestinal epithelial barrier in health and disease. Monogr. Pathol. 31, 306-324 (1990).

4. Lievin-Le Moal, V. \& Servin, A. L. The front line of enteric host defense against unwelcome intrusion of harmful microorganisms: mucins, antimicrobial peptides, and microbiota. Clin. Microbiol. Rev. 19, 315-337 (2006).

5. MacDonald, T. T. The mucosal immune system. Parasite Immunol. 25, 235-246 (2003).

6. Neutra, M. R., Mantis, N. J. \& Kraehenbuhl, J. P. Collaboration of epithelial cells with organized mucosal lymphoid tissues. Nat. Immunol. 2, 1004-1009 (2001).

7. Owen, R. L. Uptake and transport of intestinal macromolecules and microorganisms by $\mathrm{M}$ cells in Peyer's patches--a personal and historical perspective. Semin. Immunol. 11, 157-163 (1999).

8. Vetvicka, V., Tlaskalova-Hogenova, H., Fornusek, L., Rihova, B. \& Holan, V. Membrane and functional characterization of lymphoid and macrophage populations of Peyer's patches from adult and aged mice. Immunology 62, 39-43 (1987)

9. Reynolds, J. D. Peyer's patches and the early development of B lymphocytes. Curr. Top. Microbiol. Immunol. 135, 43-56 (1987).

10. Iwasaki, A. \& Kelsall, B. L. Freshly isolated Peyer's patch, but not spleen, dendritic cells produce interleukin 10 and induce the differentiation of $\mathrm{T}$ helper type 2 cells. J. Exp. Med. 190, 229-239 (1999).

11. Kelsall, B. L. \& Strober, W. Distinct populations of dendritic cells are present in the subepithelial dome and T cell regions of the murine Peyer's patch. J. Exp. Med. 183, 237-247 (1996).

12. Kraehenbuhl, J. P. \& Neutra, M. R. Epithelial M cells: differentiation and function. Annu. Rev. Cell Dev. Biol. 16, 301-332 (2000).

13. Terahara, K. et al. Comprehensive gene expression profiling of Peyer's patch $\mathrm{M}$ cells, villous $\mathrm{M}$-like cells, and intestinal epithelial cells. J. Immunol. 180, 7840-7846 (2008). 
14. Hase, K. et al. Uptake through glycoprotein 2 of $\mathrm{FimH}(+)$ bacteria by M cells initiates mucosal immune response. Nature 462, 226-230 (2009).

15. Nakato, G. et al. Cutting Edge: brucella abortus exploits a cellular prion protein on intestinal M cells as an invasive receptor. J. Immunol. 189, 1540-1544 (2012).

16. Sato, S. et al. Transcription factor Spi-B-dependent and -independent pathways for the development of Peyer's patch M cells. Mucosal Immunol. 6, 838-846 (2013).

17. Kanaya, T. et al. The Ets transcription factor Spi-B is essential for the differentiation of intestinal microfold cells. Nat. Immunol. 13, 729-736 (2012).

18. de Lau, W. et al. Peyer's patch M cells derived from $\operatorname{Lgr5}(+)$ stem cells require SpiB and are induced by RankL in cultured 'miniguts'. Mol. Cell Biol. 32, 3639-3647 (2012).

19. Utans, U., Arceci, R. J., Yamashita, Y. \& Russell, M. E. Cloning and characterization of allograft inflammatory factor-1: a novel macrophage factor identified in rat cardiac allografts with chronic rejection. J. Clin. Invest. 95, 2954-2962 (1995)

20. Mishima, T. et al. Allograft inflammatory factor-1 augments macrophage phagocytotic activity and accelerates the progression of atherosclerosis in ApoE ${ }^{-1-}$ mice. Int. J. Mol. Med. 21, 181-187 (2008).

21. Ohsawa, K., Imai, Y., Kanazawa, H., Sasaki, Y. \& Kohsaka, S. Involvement of Ibal in membrane ruffling and phagocytosis of macrophages/microglia. J. Cell Sci. 113, 3073-3084 (2000).

22. Ohsawa, K., Imai, Y., Sasaki, Y. \& Kohsaka, S. Microglia/macrophage-specific protein Ibal binds to fimbrin and enhances its actin-bundling activity. $J$. Neurochem. 88, 844-856 (2004).

23. Autieri, M. V., Kelemen, S. E. \& Wendt, K. W. AIF-1 is an actin-polymerizing and Racl-activating protein that promotes vascular smooth muscle cell migration. Circ. Res. 92, 1107-1114 (2003).

24. Knoop, K. A. et al. RANKL is necessary and sufficient to initiate development of antigen-sampling $\mathrm{M}$ cells in the intestinal epithelium. J. Immunol. 183, 5738-5747 (2009)

25. Casas, I. A. \& Dobrogosz, W. J. Validation of the probiotic concept: Lactobacillus reuteri Confers broad-spectrum protection against disease in humans and animals. Microbial Ecology in Health \& Disease 12, 247-285 (2000).

26. Walter, J. Ecological role of lactobacilli in the gastrointestinal tract: implications for fundamental and biomedical research. Appl. Environ. Microbiol. 74, 4985-4996 (2008).

27. Dramsi, S. \& Cossart, P. Intracellular pathogens and the actin cytoskeleton. Annu. Rev. Cell Dev. Biol. 14, 137-166 (1998).

28. Reis, R. S. \& Horn, F. Enteropathogenic Escherichia coli, Samonella, Shigella and Yersinia: cellular aspects of host-bacteria interactions in enteric diseases. Gut Pathog. 2, 8 (2010).

29. Kanazawa, H., Ohsawa, K., Sasaki, Y., Kohsaka, S. \& Imai, Y. Macrophage/ microglia-specific protein Ibal enhances membrane ruffling and Rac activation via phospholipase C-gamma -dependent pathway. J. Biol. Chem. 277, 20026-20032 (2002).

30. Rios, D. et al. Antigen sampling by intestinal M cells is the principal pathway initiating mucosal IgA production to commensal enteric bacteria. Mucosal Immunol. 9, 907-916 (2016)

31. Isberg, R. R. \& Leong, J. M. Multiple beta 1 chain integrins are receptors for invasin, a protein that promotes bacterial penetration into mammalian cells Cell 60, 861-871 (1990).

32. Grutzkau, A., Hanski, C., Hahn, H. \& Riecken, E. O. Involvement of M cells in the bacterial invasion of Peyer's patches: a common mechanism shared by Yersinia enterocolitica and other enteroinvasive bacteria. Gut 31, 1011-1015 (1990).

33. Autenrieth, I. B. \& Firsching, R. Penetration of M cells and destruction of Peyer's patches by Yersinia enterocolitica: an ultrastructural and histological study. J. Med. Microbiol. 44, 285-294 (1996).

34. Clark, M. A., Hirst, B. H. \& Jepson, M. A. M-cell surface beta1 integrin expression and invasin-mediated targeting of Yersinia pseudotuberculosis to mouse Peyer's patch M cells. Infect. Immun. 66, 1237-1243 (1998).

35. Imai, Y., Ibata, I., Ito, D., Ohsawa, K. \& Kohsaka, S. A novel gene ibal in the major histocompatibility complex class III region encoding an EF hand protein expressed in a monocytic lineage. Biochem. Biophys. Res. Commun. 224 855-862 (1996)

36. Casimiro, I., Chinnasamy, P. \& Sibinga, N. E. Genetic inactivation of the allograft inflammatory factor-1 locus. Genesis 51, 734-740 (2013).

37. Tsubata, Y. et al. Expression of allograft inflammatory factor-1 in kidneys: a novel molecular component of podocyte. Kidney Int. 70, 1948-1954 (2006).

38. Masuda, T. et al. IRF8 is a critical transcription factor for transforming microglia into a reactive phenotype. Cell Rep. 1, 334-340 (2012).

39. Minten, C., Terry, R., Deffrasnes, C., King, N. J. \& Campbell, I. L. IFN regulatory factor 8 is a key constitutive determinant of the morphological and molecular properties of microglia in the CNS. PLoS ONE 7, e49851 (2012).

40. Sibinga, N. E., Feinberg, M. W., Yang, H., Werner, F. \& Jain, M. K. Macrophage-restricted and interferon gamma-inducible expression of the allograft inflammatory factor-1 gene requires Pu.1. J. Biol. Chem. 277, 16202-16210 (2002)

41. Kierdorf, K. et al. Microglia emerge from erythromyeloid precursors via Pu.1- and Irf8-dependent pathways. Nat. Neurosci. 16, 273-280 (2013).

42. Schluesener, H. J., Seid, K., Kretzschmar, J. \& Meyermann, R. Allograftinflammatory factor-1 in rat experimental autoimmune encephalomyelitis, neuritis, and uveitis: expression by activated macrophages and microglial cells. Glia 24, 244-251 (1998).

43. Pawlik, A. et al. Association of allograft inflammatory factor-1 gene polymorphism with rheumatoid arthritis. Tissue Antigens 72, 171-175 (2008)

44. Rochereau, N. et al. Dectin-1 is essential for reverse transcytosis of glycosylated SIgA-antigen complexes by intestinal M cells. PLoS Biol. 11, e1001658 (2013).

45. Niess, J. H. et al. CX3CR1-mediated dendritic cell access to the intestinal lumen and bacterial clearance. Science 307, 254-258 (2005).

46. Vallon-Eberhard, A., Landsman, L., Yogev, N., Verrier, B. \& Jung, S. Transepithelial pathogen uptake into the small intestinal lamina propria. J. Immunol. 176, 2465-2469 (2006)

47. Ley, K., Laudanna, C., Cybulsky, M. I. \& Nourshargh, S. Getting to the site of inflammation: the leukocyte adhesion cascade updated. Nat. Rev. Immunol. 7, 678-689 (2007)

48. Tian, Y. \& Autieri, M. V. Cytokine expression and AIF-1-mediated activation of Rac2 in vascular smooth muscle cells: a role for Rac2 in VSMC activation. Am J. Physiol. Cell Physiol. 292, C841-C849 (2007).

49. Gulig, P. A. \& Curtiss, 3rd R. Plasmid-associated virulence of Salmonella typhimurium. Infect. Immun. 55, 2891-2901 (1987).

50. Gulig, P. A., Doyle, T. J., Hughes, J. A. \& Matsui, H. Analysis of host cells associated with the Spv-mediated increased intracellular growth rate of Salmonella typhimurium in mice. Infect. Immun. 66, 2471-2485 (1998).

51. Datsenko, K. A. \& Wanner, B. L. One-step inactivation of chromosomal genes in Escherichia coli K-12 using PCR products. Proc. Natl Acad. Sci. USA 97, 6640-6645 (2000).

\section{Acknowledgements}

We thank Dr M. Celeste Simon and Dr Hidenori Matsui for providing Spib ${ }^{-1-}$ mice and a series of Salmonella enterica serovar Typhimurium strains, respectively. We also thank Dr Hiroshi Sagara for help with the electron microscopic analyses, Mariko Kamioka for help with the bone-marrow transfer experiments, and Jun Yoshida, Yoshie Tokura, Akemi Arakawa, Chizuru Tsuzuki and Ayae Nishiyama for their technical assistance. This work was supported by grants from the Ministry of Education, Science, Sports and Technology of Japan (Grant-in Aid for Challenging Exploratory Research (23659199 to S.S.), for Young Scientists B (25860353 to S.S.), for Scientific Research (C) (16K08836 to S.S.), and for Scientific Research (S) (23229004 to H.K.)); the Global Center of Excellence Program of the Center of Education and Research for Advanced Genome-based Medicine (to H.K.); and the Core Research for Evolutional Science and Technology Program of the Japan Science and Technology Agency (to H.K.)

\section{Author contributions}

S.Ki. and S.S. designed the overall research, performed experiments, and wrote the manuscript; S.Ka. supported the infection experiments; and S.U. and S.Ko. established Aif1-deficient mice. S.N. analysed and discussed the data, and H.K. supervised the project and wrote the manuscript.

\section{Additional information}

Supplementary Information accompanies this paper at http://www.nature.com/ naturecommunications

Competing financial interests: The authors declare no competing financial interests

Reprints and permission information is available online at http://npg.nature.com/ reprintsandpermissions/

How to cite this article: Kishikawa, S. et al. Allograft inflammatory factor 1 is a regulator of transcytosis in M cells. Nat. Commun. 8, 14509 doi: 10.1038/ncomms14509 (2017).

Publisher's note: Springer Nature remains neutral with regard to jurisdictional claims in published maps and institutional affiliations.

This work is licensed under a Creative Commons Attribution 4.0 International License. The images or other third party material in this article are included in the article's Creative Commons license, unless indicated otherwise in the credit line; if the material is not included under the Creative Commons license users will need to obtain permission from the license holder to reproduce the material. To view a copy of this license, visit http://creativecommons.org/licenses/by/4.0/ 